\title{
Design of an on-line check device for safety valve
}

\author{
Yan Zhipeng ${ }^{1}$,Li Qing ${ }^{1, a}$,Jin Muda ${ }^{2}$, Zhong Haijian $^{2}$ \\ ${ }^{1}$ China Jiliang University, Hangzhou, China \\ ${ }^{2}$ Zhejiang Special Equipment Inspection and Research Institute, Hangzhou, China
}

\begin{abstract}
As the main pressure relief component of the tanker, the spring type safety valve is indispensable for its periodic verification. An on-line check device is designed for the check problem of the safety valve. The check device is divided into mechanical structure, program control, motor drive, tension detection, displacement measurement, upper computer, etc., and the safety valve is verified according to the safety valve check principle and the determination method. Compared with the check device that uses the hydraulic system to provide the pulling force, the device has the advantages of simple operation, high speed, convenient disassembly, strong applicability, and the like, and has certain market application prospects.
\end{abstract}

\section{Introduction}

At present, China's special gases such as propane and propylene are mainly transported by tankers [1]. As the main pressure relief component of the tanker, the safety valve will change its setting pressure and sealing performance under the action of pressure, temperature and physical and chemical factors during operation. As a result, the safety valve cannot be opened according to the set pressure, which affects safe production, so regular calibration of safety valves is essential [2]. The method of checking the safety valve is divided into two kinds of off-line check and on-line check[3]. The method of off-line checking of the safety valve is to remove the safety valve from the device and send it to the check table. Some of the disassembly, installation and transportation of the safety valves are more difficult. There are also problems such as long check time and high comprehensive cost $[4,5]$. At present, the on-line check technology of safety valve is developing rapidly. This paper proposes a new type of safety valve online check device, which is convenient and simple to operate.

\section{Online checkout}

\subsection{Online check principle}

Figure 1 shows the safety valve check schematic. An additional pulling force $\mathrm{F}$ is applied to the safety valve bonnet. When the additional force $\mathrm{F}$ is gradually increased until the pressing force between the safety valve disc and the tank body is equal to zero. The safety valve seal begins to lose and will enter the open state [6], at which time the sum of the medium force and the additional tension just overcomes the spring preload.

$$
\mathrm{F}_{\mathrm{d}}=\mathrm{P}_{0} \mathrm{~S}+\mathrm{F}
$$

The setting pressure of safety valve can be calculated by formula (1).

$$
\mathrm{P}_{\mathrm{s}}=\mathrm{P}_{0}+\mathrm{F} / \mathrm{S}
$$

In formula (1), (2) and figure $1, \mathrm{~F}_{\mathrm{d}}$ is a spring pretightening force, $\mathrm{S}$ is the effective area on the valve disc, $P_{0}$ is the pressure of the medium in the system, $P_{s}$ is the setting pressure of the safety valve, and $\mathrm{F}$ is used as an upwards additional pull force on the valve valve of the safety valve.

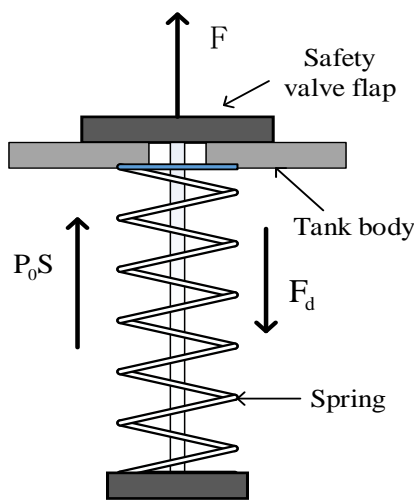

Figure 1. Safety valve check principle 


\subsection{Safety valve judgment method.}

The additional tension curve feature point method is that the additional pulling force provided by the online check device is gradually increased. When the sum of the additional tensile force and the medium force of the system itself is equal to the spring preloading force, the valve flap is slightly raised. As the effective area of the medium increases instantaneously, the force of the medium is instantaneously increased, and the additional external force is instantaneously reduced. Thus, the characteristic point A appears as shown in Figure 2, and point A is the safety valve micro-start point. During the safety valve returning process, the additional pulling force provided by the on-line check device is gradually reduced. When the valve flap reaches the closing point, the medium acting area of the system is instantaneously reduced due to the instantaneous reduction of the working area of the medium. In order to ensure the balance of the valve flap, the additional pulling force will rebound instantaneously, resulting in a return point B. In the cold state verification, there is no force of the medium. At this time, the additional tension will appear as two characteristic points of $\mathrm{C}$ and $\mathrm{D}$ as shown in Figure 3.

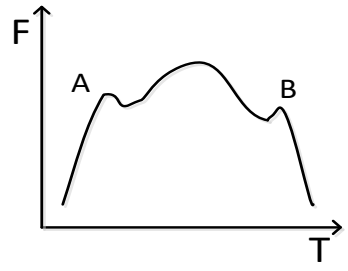

Figure 2. Additional tensile force curve in hot stat

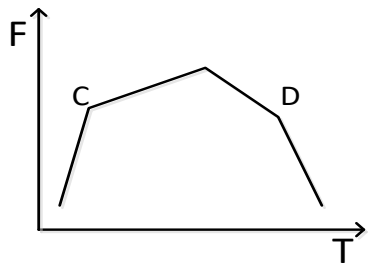

Figure 3. Additional tension curve in cold state

\section{Check device design}

\subsection{The structure of the check device}

As shown in Figure 4, the structure of the check device is shown. The check device provides torque from the stepper motor. The torque is amplified by the reducer. The reducer is connected through the coupling sleeve and the screw nut. The rotation of the motor drives the screw nut to rotate, the screw nut is placed on the thrust bearing, and the screw nut rotates to drive the screw rod to move upwards linearly, and the screw rod is prevented from rotating by the mechanical structure, and the lower end of the screw rod protrudes a magnet through the gasket. The movement of the screw moves the magnet, and the movement of the magnet corresponds to the change of the voltage outputted by the Hall sensor placed below, thereby measuring the displacement of the screw, and the tension sensor under the gasket is connected with the screw. The lower end of the tension sensor is connected to the quick connector, and the quick connector is connected to the safety valve. The height and tension of the safety valve opening can be measured during the opening of the safety valve.

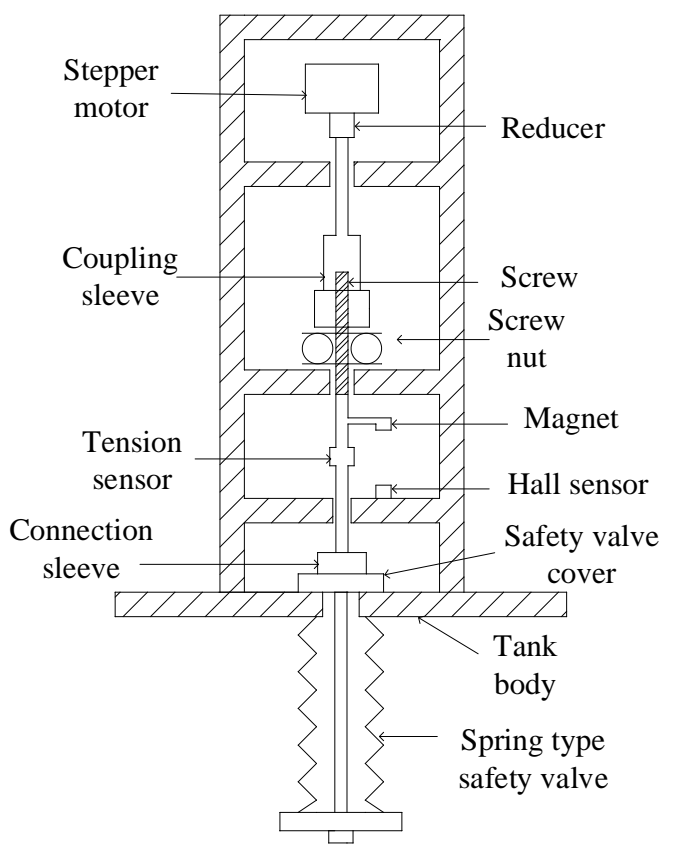

Figure 4. Structure of the check device

\subsection{Hardware circuit design}

The hardware circuit system mainly realizes the driving and control of the stepping motor, the sensor data acquisition and transmission, and the main hardware includes the motor drive module, the sensor signal acquisition and amplification, the power supply circuit and the wireless module. A general block diagram of the hardware design is given in Figure 5.

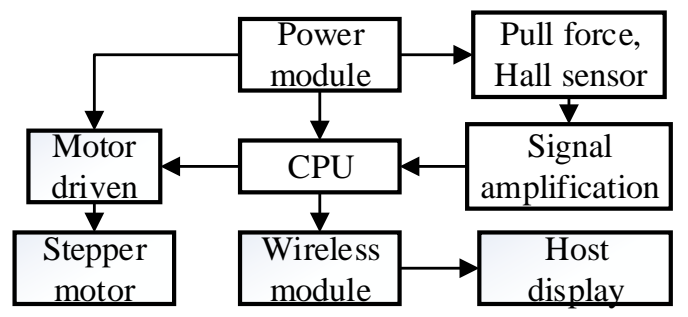

Figure 5. Hardware design block diagram

\section{Test and result analysis}

\subsection{Displacement calibration and testing}

The magnet is calibrated from $28 \mathrm{~mm}$ to $40 \mathrm{~mm}$ above the Hall sensor, and then modeling and analysis is performed to obtain the relationship between the output voltage and 
the displacement of the Hall sensor. The accurate displacement can be calculated from the output voltage of the Hall sensor [7]. In the actual measurement, the $28 \mathrm{~mm}$ above the Hall sensor is used as the starting point, and then the measurement is made within $12 \mathrm{~mm}$ above the starting point. The comparison between the measured value and the actual value is shown in Table 1.

Table 1. Displacement test results

\begin{tabular}{|c|c|c|}
\hline $\begin{array}{c}\text { Actual } \\
\text { displacement } \\
{[\mathrm{mm}]}\end{array}$ & $\begin{array}{c}\text { Experimental } \\
\text { measurement } \\
{[\mathrm{mm}]}\end{array}$ & Error[mm] \\
\hline 1.000 & 1.014 & 0.014 \\
\hline 2.000 & 2.022 & 0.022 \\
\hline 3.000 & 2.981 & -0.019 \\
\hline 4.000 & 3.993 & -0.007 \\
\hline 5.000 & 4.974 & -0.036 \\
\hline 6.000 & 6.052 & 0.052 \\
\hline 7.000 & 7.087 & 0.087 \\
\hline 8.000 & 7.895 & -0.115 \\
\hline 9.000 & 9.064 & 0.064 \\
\hline 10.000 & 10.135 & 0.135 \\
\hline 11.000 & 11.158 & 0.158 \\
\hline 12.000 & 11.964 & -0.036 \\
\hline
\end{tabular}

Through the experimental data in Table 1, it can be concluded that the experimental results are relatively stable, the experimental error is small. The test error is within $0.2 \mathrm{~mm}$, and the displacement can be measured well.

\subsection{Safety valve test}

In the system test, the two types of safety valves of type A411F-2.5 and A412F-2.5 were tested, and the full-open safety valve with the diameter of DN $50 \mathrm{~mm}$ and the effective area of the valve $1963.5 \mathrm{~mm}^{2}$ was off-line verified. Figure 6 is a graph showing the measured tensile force and displacement of the A411F-2.5 type safety valve. The blue curve is the tensile force curve, the tensile force is in $\mathrm{kg}$, the red curve is the displacement curve, and the displacement is in $\mathrm{mm}$.

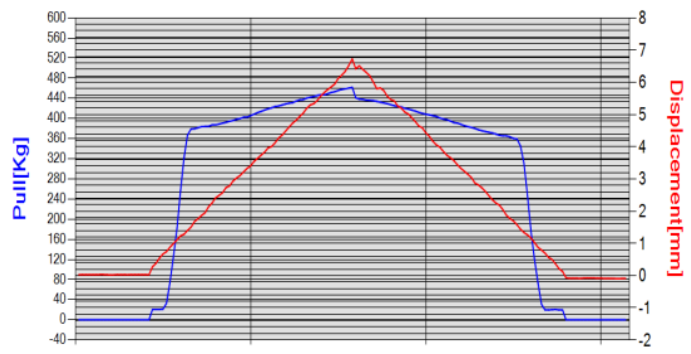

Figure 6. Tension and displacement curve

It can be clearly seen from Figure 6 that the safety valve is opened and closed at a constant speed. When the valve flap is slightly opened, there will be an obvious additional pull point of the pull force, and the pull value of the inflection point is obtained, and the set pressure of the safety valve can be calculated.

Table 2. Set pressure test results

\begin{tabular}{|l|l|l|l|l|}
\hline $\begin{array}{l}\text { Safety } \\
\text { valve } \\
\text { model }\end{array}$ & $\begin{array}{l}\text { Pull } \\
\text { value } \\
{[\mathrm{N}]}\end{array}$ & $\begin{array}{l}\text { measured } \\
\text { value } \\
{[\mathrm{MPa}]}\end{array}$ & $\begin{array}{l}\text { Actual } \\
\text { value } \\
{[\mathrm{MPa}]}\end{array}$ & $\begin{array}{l}\text { Error } \\
{[\mathrm{MPa}]}\end{array}$ \\
\hline $\begin{array}{c}\text { A411F } \\
-2.5\end{array}$ & 3676 & 1.872 & 1.9 & -0.028 \\
\cline { 2 - 5 } & 3781 & 1.925 & 1.9 & 0.025 \\
\cline { 2 - 5 } & 3810 & 1.940 & 1.9 & 0.040 \\
\hline \multirow{2}{*}{$\begin{array}{l}\text { A412F } \\
-2.5\end{array}$} & 4065 & 2.070 & 2.1 & -0.035 \\
\cline { 2 - 5 } & 4184 & 2.131 & 2.1 & 0.031 \\
\cline { 2 - 5 } & 4217 & 2.148 & 2.1 & 0.048 \\
\hline
\end{tabular}

From Table 2, it can be calculated that the average error of the three-dimensional calibration of the A411F-2.5 safety valve is $0.031 \mathrm{MPa}$, and the average error is $1.6 \%$. The average error of the three-dimensional calibration of the A412F-2.5 safety valve is $0.038 \mathrm{MPa}$, and the average error is $1.8 \%$. The error is small and meets the error requirements.

\section{Conclusion}

The system designs and implements a new on-line check device for safety valve, which can accurately measure the opening height of the safety valve and verify the setting pressure of the safety valve. It makes the safety valve check more convenient, improves the scientificity of safety valve verification, reduces the workload of personnel, saves costs, and has certain market prospects.

\section{References}

1. Ma Cunxi. Research on Risk Identification and Evaluation of Butadiene Tanker Road Transportation. D. (2012)

2. Min Jiafeng. Research on key technology of safety valve online verification. D. (2014)

3. Liu Hailang. Research and implementation of safety valve online calibration system. D. (2010)

4. Li Bingling. Development of on-line verification system for safety valves. J. Chemical Equipment Technology, 33 (02): 58-61 (2012)

5. Cai Chuangming, Liu Weizhong, Qiu Yanfei, Wang Youhong. Development of JX-2006 Safety Valve Online Calibrato. J. (08):103-105 (2006)

6. Wang Aitao, Zhu Haiqing. On-line verification system of safety valve. J. Light Industry Machinery, 34(05): 59-62 (2016)

7. Tian Wei, Liu Zongtian. Piecewise straight line fitting of least squares method. J. Computer Science, (S1): 482-484 (2012) 